PROCEEDINGS OF THE

AMERICAN MATHEMATICAL SOCIETY

Volume 130, Number 3, Pages 707-713

S 0002-9939(01)06214-1

Article electronically published on June 20, 2001

\title{
CANONICAL DIVISORS IN WEIGHTED BERGMAN SPACES
}

\author{
RACHEL J. WEIR
}

(Communicated by Juha M. Heinonen)

\begin{abstract}
Canonical divisors in Bergman spaces can be found as solutions of extremal problems. We derive a formula for certain extremal functions in the weighted Bergman spaces $A_{\alpha}^{p}$ for $\alpha>-1$ and $1 \leq p<\infty$. This leads to a study of the zeros of a specific family of hypergeometric functions.
\end{abstract}

\section{INTRODUCTION}

The Bergman space $A^{p}$ is the space of functions analytic in the unit disk $\mathbb{D}$ for which

$$
\|f\|_{p}=\left(\int_{\mathbb{D}}|f(z)|^{p} d \sigma(z)\right)^{\frac{1}{p}}<\infty
$$

where $d \sigma$ is normalized area measure on the disk and $0<p<\infty$. Given an $A^{2}$ zeroset $\left\{\zeta_{j}\right\}$ with $\zeta_{j} \neq 0$, Hedenmalm [2] showed that the solution $G$ of the extremal problem

$$
\sup \left\{\operatorname{Re} f(0): f \in A^{2}, f\left(\left\{\zeta_{j}\right\}\right)=0,\|f\|_{2}=1\right\}
$$

is a contractive divisor. In other words, $G$ vanishes precisely on the set $\left\{\zeta_{j}\right\}$ and, for any function $f$ in $A^{2}$ which also vanishes on this set, $f / G \in A^{2}$ and $\|f / G\|_{2} \leq\|f\|_{2}$. Duren, Khavinson, Shapiro and Sundberg [1] obtained the analogous results for $A^{p}$.

The weighted Bergman space $A_{\alpha}^{p}$ is the space of functions analytic in the disk for which

$$
\|f\|_{p, \alpha}=\left(\int_{\mathbb{D}}|f(z)|^{p} w_{\alpha}(z) d \sigma(z)\right)^{\frac{1}{p}}<\infty
$$

where $w_{\alpha}(z)=(\alpha+1)\left(1-|z|^{2}\right)^{\alpha}, \alpha>-1$ and $0<p<\infty$. Shimorin [6], 7] proved that for $-1<\alpha \leq 1$ the analogous extremal functions are contractive divisors in $A_{\alpha}^{2}$. The present paper considers these canonical divisors in the weighted Bergman space $A_{\alpha}^{p}$. For simplicity, we restrict ourselves to the case $1 \leq p<\infty$. We will derive an explicit formula for the extremal function when the zero-set consists of a single point. The formula involves hypergeometric functions and is valid when these functions are nonvanishing in a given disk. We will discuss how the location

Received by the editors August 22, 2000.

2000 Mathematics Subject Classification. Primary 32A36; Secondary 33C05.

The contents of this paper forms a part of the author's doctoral dissertation at the University of Michigan, written under the direction of Professor Peter Duren, whose support and guidance the author gratefully acknowledges. 
of the zeros of these hypergeometric functions depends on $p$ and $\alpha$, giving both analytic and numerical results.

\section{Extremal functions in $A_{\alpha}^{p}$}

A sequence of points $\left\{\zeta_{j}\right\}$ in the unit disk is an $A_{\alpha}^{p}$ zero-set if there is a function in $A_{\alpha}^{p}$ which vanishes precisely on this set. In other words, the function vanishes only at the points in the sequence and the multiplicity of each zero is determined by the number of times a particular point appears in the sequence. For convenience, we assume that $\zeta_{j} \neq 0$ for $j=1,2, \ldots$. We let $N_{\alpha}^{p}$ denote the set of functions in $A_{\alpha}^{p}$ which vanish on a given $A_{\alpha}^{p}$ zero-set. We can then pose the extremal problem

$$
\sup \left\{\operatorname{Re} f(0): f \in N_{\alpha}^{p},\|f\|_{p, \alpha}=1\right\} .
$$

The existence and uniqueness of the extremal function can be established by generalizing the methods used in [1].

The following proposition gives a sufficient condition for a given function to be extremal.

Proposition 2.1. Suppose $g \in N_{\alpha}^{p}$ satisfies

$$
\int_{\mathbb{D}}|g(z)|^{p-2} \overline{g(z)} h(z) w_{\alpha}(z) d \sigma(z)=0
$$

for all $h \in N_{\alpha}^{p}$ with $h(0)=0$. Then $g$ is a constant multiple of the extremal function corresponding to the given zero-set.

To prove this we use the following lemma.

Lemma 2.2. For $1 \leq p<\infty$, let $M$ be a closed subspace of $L^{p}\left(\mathbb{D}, w_{\alpha} d \sigma\right)$. If $g \in L^{p}\left(\mathbb{D}, w_{\alpha} d \sigma\right)$ satisfies (2.1), then $\|g\|_{p, \alpha}=\operatorname{dist}(g, M)$.

The proofs of these two results are obtained by modifying the arguments given in [1] and will be omitted.

\section{The MAIN RESUlt}

We now suppose that our given zero-set consists of a single point. This case is of importance when we consider whether the extremal function has extraneous zeros. Since we wish to divide by our extremal function $G$, it is essential that $G$ have no zeros in the unit disk other than those prescribed. Showing that the single-point extremal function has no extraneous zeros is a key step in proving the same for a general extremal function (see, for instance, [1, p. 45).

Before stating the result, we recall that the Gauss hypergeometric function is defined as

$$
F(a, b ; c ; z)=\sum_{n=0}^{\infty} \frac{(a)_{n}(b)_{n}}{(c)_{n}} \frac{z^{n}}{n !}
$$

where $(x)_{n}=\Gamma(x+n) / \Gamma(x)$ is Pochhammer's symbol. This series converges for all $z$ in the unit disk (see [5] for details). 
Theorem 3.1. Suppose $\alpha>-1$ and $1 \leq p<\infty$. Let $\beta \in \mathbb{D} \backslash\{0\}$. Then in $A_{\alpha}^{p}$ the extremal function $G_{\beta}(z)$ corresponding to the zero-set $\{\beta\}$ is

$$
G_{\beta}(z)=\frac{\bar{\beta}}{|\beta|} \frac{\beta-z}{1-\bar{\beta} z}\left[\frac{F\left(-\alpha-1, \frac{p}{2} ; \frac{p}{2}+1 ; \bar{\beta} \frac{\beta-z}{1-\bar{\beta} z}\right)^{2}}{(\alpha+1) B\left(\alpha+1, \frac{p}{2}+1\right) F\left(-\alpha-1, \frac{p}{2} ; \frac{p}{2}+1 ;|\beta|^{2}\right)}\right]^{\frac{1}{p}}
$$

whenever $F\left(-\alpha-1, \frac{p}{2} ; \frac{p}{2}+1 ; w\right) \neq 0$ in the disk $\{|w|<|\beta|\}$.

Proof. Let $\beta$ be a point in the unit disk, not the origin. By Proposition 2.1, it is enough to find a function $g$ vanishing at $\beta$ and satisfying (2.1), normalized by $\|g\|_{p, \alpha}=1$ and $g(0)>0$.

For $h \in A_{\alpha}^{p}$, we can write the orthogonality condition (2.1) in the form

$$
\int_{\mathbb{D}}|g(z)|^{p-2} \overline{g(z)}\left(\frac{\beta-z}{1-\bar{\beta} z}\right) z h(z)(\alpha+1)\left(1-|z|^{2}\right)^{\alpha} d \sigma(z)=0 .
$$

Since $g$ must have the prescribed zero at $\beta$, we may write $g(z)=\varphi_{\beta}(z) f(z)$, where $f$ is analytic and nonvanishing in the unit disk and $\varphi_{\beta}(z)=(\beta-z) /(1-\bar{\beta} z)$. Thus,

$$
\int_{\mathbb{D}}\left|\varphi_{\beta}(z)\right|^{p-2}|f(z)|^{p-2} \overline{\varphi_{\beta}(z) f(z)} \varphi_{\beta}(z) z h(z)(\alpha+1)\left(1-|z|^{2}\right)^{\alpha} d \sigma(z)=0 .
$$

Letting $w=\varphi_{\beta}(z)$, we require that

$$
\int_{\mathbb{D}}|w|^{p} \varphi_{\beta}(w)\left(1-|w|^{2}\right)^{\alpha} \overline{\Phi(w)} \Psi(w) d \sigma(w)=0,
$$

where

$$
\Phi(w)=f\left(\varphi_{\beta}(w)\right)^{\frac{p}{2}} \frac{\left(1-|\beta|^{2}\right)^{\alpha+1}}{(1-\bar{\beta} w)^{\alpha+2}}
$$

and

$$
\Psi(w)=f\left(\varphi_{\beta}(w)\right)^{\frac{p}{2}-1} h\left(\varphi_{\beta}(w)\right) \frac{\left(1-|\beta|^{2}\right)}{(1-\bar{\beta} w)^{\alpha+2}} .
$$

As $\Psi$ is arbitrary, we set $\Psi(w)=(1-\bar{\beta} w) w^{k}$ and $\Phi(w)=\sum_{n=0}^{\infty} c_{n} w^{n}$. Thus,

$$
\overline{c_{k+1}} B\left(\frac{p}{2}+k+2, \alpha+1\right)-\beta \overline{c_{k}} B\left(\frac{p}{2}+k+1, \alpha+1\right)=0,
$$

where $B(x, y)$ is the beta function. Therefore

$$
c_{k}=\bar{\beta} \frac{B\left(\frac{p}{2}+k, \alpha+1\right)}{B\left(\frac{p}{2}+k+1, \alpha+1\right)} c_{k-1}=\bar{\beta}^{k} \frac{B\left(\frac{p}{2}+1, \alpha+1\right)}{B\left(\frac{p}{2}+k+1, \alpha+1\right)} c_{0} .
$$

Solving for $f$ in the definition of $\Phi$ gives

$$
f\left(\varphi_{\beta}(w)\right)=\left[\frac{(1-\bar{\beta} w)^{\alpha+2}}{\left(1-|\beta|^{2}\right)^{\alpha+1}} \sum_{n=0}^{\infty} c_{n} w^{n}\right]^{\frac{2}{p}} .
$$

As $w=\varphi_{\beta}(z)$, inserting the formula for $c_{n}$ and taking $c_{0}=1$, we have

$$
f(z)=\left[\frac{1-|\beta|^{2}}{(1-\bar{\beta} z)^{\alpha+2}} \sum_{n=0}^{\infty}(\bar{\beta})^{n} \frac{B\left(\frac{p}{2}+1, \alpha+1\right)}{B\left(\frac{p}{2}+n+1, \alpha+1\right)}\left(\varphi_{\beta}(z)\right)^{n}\right]^{\frac{2}{p}} .
$$


Now,

Hence

$$
\begin{aligned}
\sum_{n=0}^{\infty} \frac{B\left(\frac{p}{2}+1, \alpha+1\right)}{B\left(\frac{p}{2}+n+1, \alpha+1\right)} w^{n} & =\sum_{n=0}^{\infty} \frac{\left(\frac{p}{2}+\alpha+2\right)_{n}(1)_{n}}{\left(\frac{p}{2}+1\right)_{n}} \frac{w^{n}}{n !} \\
& =F\left(\frac{p}{2}+\alpha+2,1 ; \frac{p}{2}+1 ; w\right) .
\end{aligned}
$$

$$
f(z)=\left[\frac{1-|\beta|^{2}}{(1-\bar{\beta} z)^{\alpha+2}} F\left(\frac{p}{2}+\alpha+2,1 ; \frac{p}{2}+1 ; \bar{\beta} \varphi_{\beta}(z)\right)\right]^{\frac{2}{p}} .
$$

Thus the function $g$ with the required orthogonality property is

$$
g(z)=\varphi_{\beta}(z) f(z)=\varphi_{\beta}(z)\left[\frac{1-|\beta|^{2}}{(1-\bar{\beta} z)^{\alpha+2}} F\left(\frac{p}{2}+\alpha+2,1 ; \frac{p}{2}+1 ; \bar{\beta} \varphi_{\beta}(z)\right)\right]^{\frac{2}{p}} .
$$

Finally, we normalize $g$ to give our extremal function $G_{\beta}$ which satisfies $\left\|G_{\beta}\right\|_{p, \alpha}=1$ and $G_{\beta}(0)>0$. Hence $G_{\beta}(z)$ has the form

$$
\frac{\bar{\beta} \varphi_{\beta}(z)}{|\beta|}\left[\frac{\left(1-|\beta|^{2}\right)^{\alpha+2} F\left(\frac{p}{2}+\alpha+2,1 ; \frac{p}{2}+1 ; \bar{\beta} \varphi_{\beta}(z)\right)^{2}}{(\alpha+1) B\left(\frac{p}{2}+1, \alpha+1\right)(1-\bar{\beta} z)^{2(\alpha+2)} F\left(\frac{p}{2}+\alpha+2,1 ; \frac{p}{2}+1 ;|\beta|^{2}\right)}\right]^{\frac{1}{p}} .
$$

To get our formula into the form (3.1), we use the hypergeometric identity (see, for instance, [5])

$$
F(a, b ; c ; z)=(1-z)^{c-a-b} F(c-a, c-b ; c ; z) .
$$

Because $\varphi_{\beta}$ is a disk automorphism, $\left|\varphi_{\beta}(z)\right| \leq 1$ for all $z$ in the closed unit disk. Therefore, since $|\beta|<1$, even for $z$ on the unit circle, the series that defines $F\left(-\alpha-1, \frac{p}{2} ; \frac{p}{2}+1 ; \bar{\beta} \varphi_{\beta}(z)\right)$ will converge. Therefore, as each of the partial sums is analytic in the closed disk, we know that $F$ itself will be analytic in the closed disk. Since by hypothesis $F$ does not vanish in the disk $\{|w|<|\beta|\}$, we see that $G_{\beta}$ is a well-defined, single-valued analytic function in the open unit disk.

\section{VAlidity of the FORMula}

We can now compare the formula in Theorem 3.1 to previously known formulas for the single-point extremal function. The first case we consider is $\alpha=0$, the unweighted Bergman space. Then

$$
F\left(-\alpha-1, \frac{p}{2} ; \frac{p}{2}+1 ; z\right)=F\left(-1, \frac{p}{2} ; \frac{p}{2}+1 ; z\right)=1-\frac{p}{p+2} z
$$

Also, $B\left(\alpha+1, \frac{p}{2}+1\right)=B\left(1, \frac{p}{2}+1\right)=2 /(p+2)$. Thus, in $A^{p}\left(=A_{0}^{p}\right)$,

$$
G_{\beta}(z)=\frac{\bar{\beta}}{|\beta|} \frac{\beta-z}{1-\bar{\beta} z}\left[\frac{\left(1+\frac{p}{2}\left(1-\bar{\beta} \frac{\beta-z}{1-\bar{\beta} z}\right)\right)^{2}}{1+\frac{p}{2}\left(1-|\beta|^{2}\right)}\right]^{\frac{1}{p}} .
$$

This is the formula found in [1].

We now look at the case $p=2$. Since $(-\alpha-1)_{n}=-(-\alpha-2)_{n+1} /(\alpha+2)$, we see by the binomial formula $(1-y)^{-a}=\sum_{n=o}^{\infty}(a)_{n} y^{n} / n$ ! that

$$
F\left(-\alpha-1, \frac{p}{2} ; \frac{p}{2}+1 ; z\right)=\frac{-1}{(\alpha+2) z}\left[(1-z)^{\alpha+2}-1\right] .
$$


Now, since $B\left(\alpha+1, \frac{p}{2}+1\right)=B(\alpha+1,2)=((\alpha+1)(\alpha+2))^{-1}$, we have

$$
G_{\beta}(z)=\sqrt{\alpha+1} \frac{1-\left(\frac{1-|\beta|^{2}}{1-\bar{\beta} z}\right)^{\alpha+2}}{\sqrt{1-\left(1-|\beta|^{2}\right)^{\alpha+2}}},
$$

which is the formula found in 3 .

Since the validity of formula (3.1) depends on the nonvanishing of the function $F\left(-\alpha-1, \frac{p}{2} ; \frac{p}{2}+1 ; z\right)$, it is important to know about the location of the zeros of this function. We first state the following result.

Theorem 4.1. For $\alpha \in\{1,2,3\}$ and $1 \leq p<\infty$, the hypergeometric function $F\left(-\alpha-1, \frac{p}{2} ; \frac{p}{2}+1 ; z\right)$ has no zeros in the unit disk.

This result follows from the proof of Proposition 2 in [4]. Note that the polynomials $(\alpha-1) P_{k}(\alpha, u), k=1,2, \ldots$, in that paper are precisely the hypergeometric polynomials $F(-k, \alpha-1 ; \alpha ; u)$.

Corollary 4.2. For $\alpha \in\{1,2,3\}$ and $1 \leq p<\infty$, the extremal function $G_{\beta}$ is given by the formula (3.1). Moreover, $G_{\beta}(z) \neq 0$ in $\mathbb{D}$ except for a simple zero at $\beta$.

The hypergeometric function $F\left(-\alpha-1, \frac{p}{2} ; \frac{p}{2}+1 ; z\right)$ is a polynomial of degree $\alpha+1$ precisely when $\alpha$ is a non-negative integer. In this case we can say something about the behavior of its zeros as $p$ tends to infinity.

Theorem 4.3. For each $\alpha=0,1,2, \ldots$, the zeros of the hypergeometric polynomial $F\left(-\alpha-1, \frac{p}{2} ; \frac{p}{2}+1 ; z\right)$ approach the point 1 as $p \rightarrow \infty$.

By noting that $F\left(-\alpha-1, \frac{p}{2} ; \frac{p}{2}+1 ; z\right) \rightarrow(1-z)^{\alpha+1}$ as $p \rightarrow \infty$, this result follows from Hurwitz' theorem.

Finally, we remark that if $F\left(-\alpha-1, \frac{p}{2} ; \frac{p}{2}+1 ; z\right)$ has any real zeros, they must lie in the interval $(1, \infty)$. This can be seen by examining the Euler integral representation

$$
F\left(-\alpha-1, \frac{p}{2} ; \frac{p}{2}+1 ; z\right)=\frac{p}{2} \int_{0}^{1} t^{\frac{p}{2}-1}(1-z t)^{\alpha+1} d t
$$

which is valid for all $p>0$ ([5], p. 47).

\section{Numerical Results}

If $\alpha$ is a positive integer, the hypergeometric function $F\left(-\alpha-1, \frac{p}{2} ; \frac{p}{2}+1 ; z\right)$ reduces to a polynomial of degree $\alpha+1$. Thus, using Mathematica, we can find the zeros of this function numerically for fixed values of $p$. Plotting these zeros, we see that they seem to lie on or near a curve which resembles a circle or lemniscate. Also, as $p$ increases, the diameter of this curve decreases and the zeros approach the point 1 as $p \rightarrow \infty$, as stated in Theorem 4.3 (See Figure 1)

When $\alpha$ is not an integer, $F\left(-\alpha-1, \frac{p}{2} ; \frac{p}{2}+1 ; z\right)$ is not a polynomial and its zeros are more difficult to compute. Instead, we let $\gamma$ denote the image of the unit circle under $F\left(-\alpha-1, \frac{p}{2} ; \frac{p}{2}+1 ; z\right)$. We can then study the winding number of $\gamma$ about the origin. If this winding number is zero, $F\left(-\alpha-1, \frac{p}{2} ; \frac{p}{2}+1 ; z\right)$ has no zeros in $\mathbb{D}$.

For $p=2$, it was shown in [3] that for $\alpha>4$ the extremal function always has extra zeros in $\mathbb{D}$, for appropriate choices of $\beta$. Also, for $\alpha<4$ the extremal function does not vanish in $\mathbb{D}$, except for a simple zero at $\beta$. So for $p=2, \alpha=4$ is the important cutoff value. Using the winding number technique, we have approximated 
the respective cutoff values of $\alpha$ for $p=1,2, \ldots, 10$. This technique is illustrated in Figure 2 As $p$ increases, this cutoff value appears to decrease. The approximate cutoff values are shown in the following table and plotted in Figure 3

\begin{tabular}{c|c|c|c|c|c|c|c|c|c|c}
$p$ & 1 & 2 & 3 & 4 & 5 & 6 & 7 & 8 & 9 & 10 \\
\hline$\alpha$ & 4.381 & 4.000 & 3.842 & 3.758 & 3.706 & 3.672 & 3.646 & 3.630 & 3.617 & 3.607
\end{tabular}

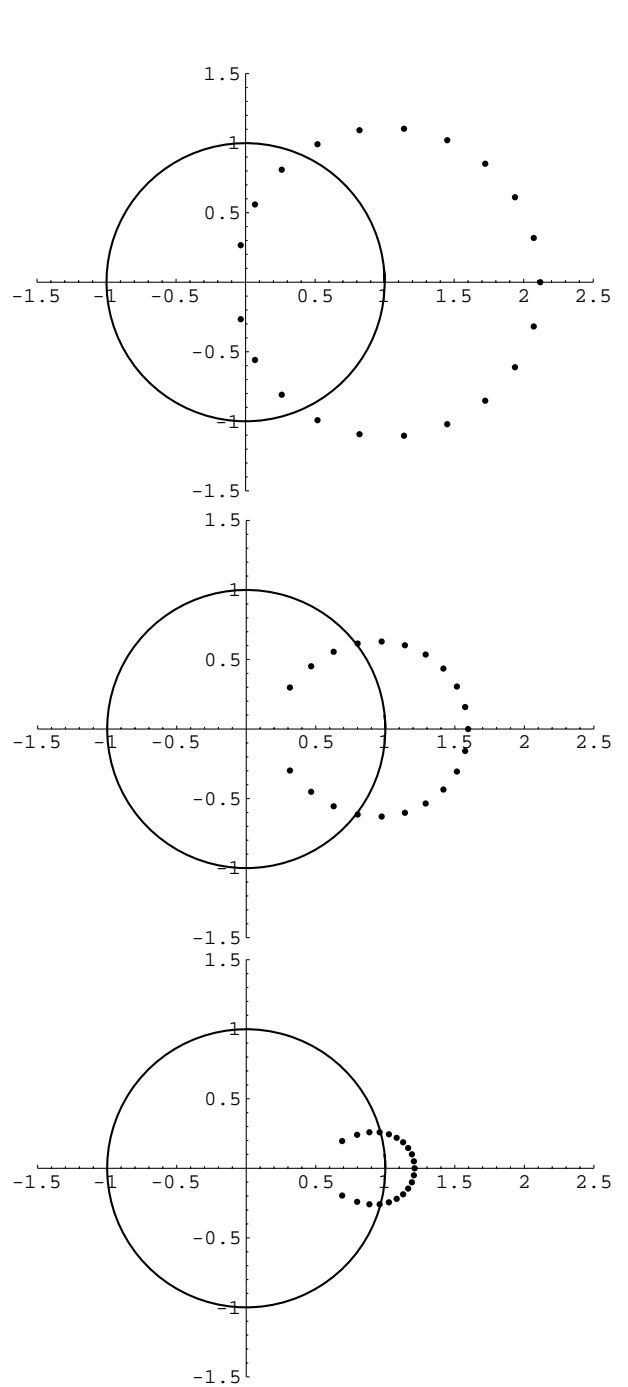

Figure 1. Zeros of

$F\left(-\alpha-1, \frac{p}{2} ; \frac{p}{2}+1 ; z\right)$, $\alpha=20, p=1,10,50$.
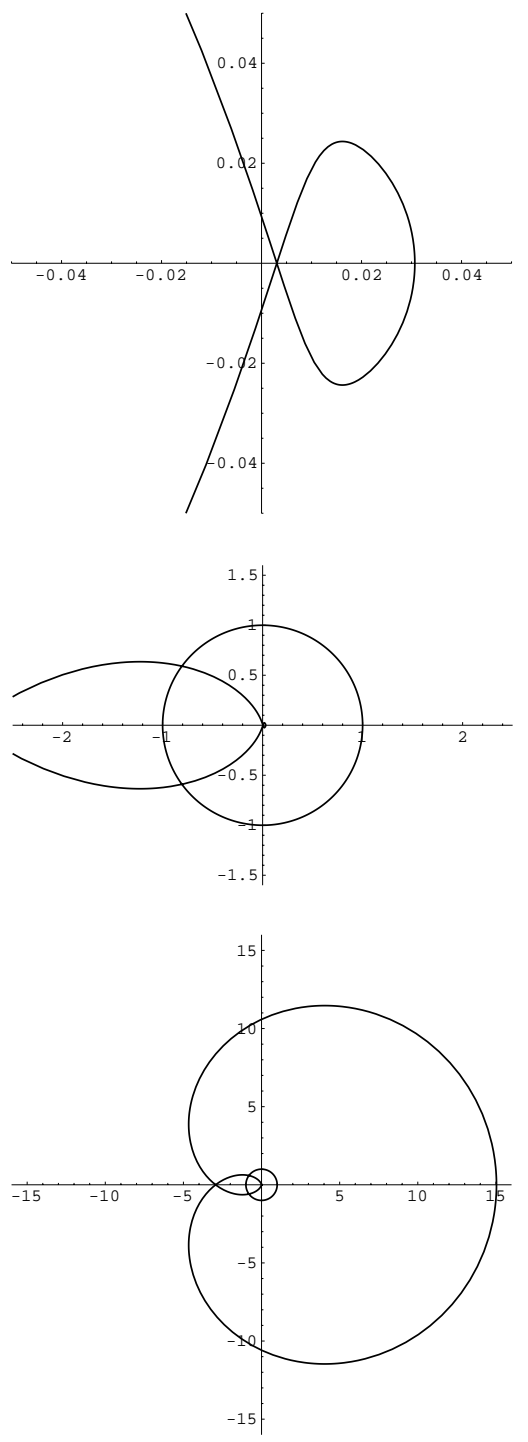

FiguRE 2. Image of the unit circle under $F\left(-\alpha-1, \frac{p}{2} ; \frac{p}{2}+1 ; z\right)$, $p=5, \alpha=3.8$; winding number about the origin $=2$. 


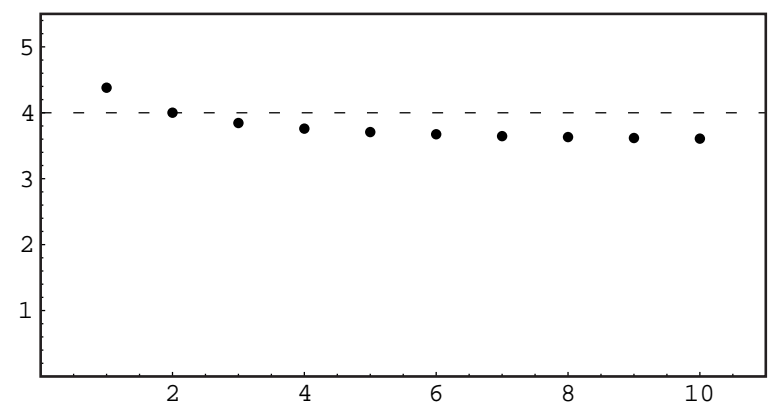

FiguRE 3. Extraneous zeros: approximate cutoff values of $\alpha$ for $p=1,2, \ldots, 10$.

\section{REFERENCES}

[1] P. Duren, D. Khavinson, H.S. Shapiro and C. Sundberg, Contractive zero-divisors in Bergman spaces, Pacific J. Math. 157 (1993), 37-56. MR 94c:30048

[2] H. Hedenmalm, A factorization theorem for square area-integrable analytic functions, J. Reine Angew. Math. 422 (1991), 45-68. MR 93c:30053

[3] H. Hedenmalm and K. Zhu, On the failure of optimal factorization for certain weighted Bergman spaces, Complex Variables Theory Appl. 19 (1992), 165-176. MR 95f:30064

[4] K.Y. Osipenko and M.I. Stessin, On optimal recovery of a holomorphic function in the unit ball of $\mathbb{C}^{n}$, Constr. Approx. 8 (1992), 141-159. MR 93d:32008

[5] E.D. Rainville, Special Functions, Macmillan, New York, 1960. MR 21:6447

[6] S.M. Shimorin, Factorization of analytic functions in weighted Bergman spaces, Algebra i Analiz 5 (1993), no. 5, 155-177 (Russian); St. Petersburg Math. J. 5 (1994), 1005-1022. MR 95j:30032

[7] - On a family of conformally invariant operators, Algebra i Analiz 7 (1995), no. 2, 133-158 (Russian); St. Petersburg Math. J. 7 (1996), 287-306. MR 96i:47010

Department of Mathematics, University of Michigan, Ann Arbor, Michigan 48109

E-mail address: rjweir@math.lsa.umich.edu

Current address: Department of Mathematics, University of Virginia, Charlottesville, Virginia 22904 